\title{
Capturing Near Atomic Resolution Snapshots of the Ribosome Assembly Process Using Direct Electron Detectors
}

\section{Joaquin Ortega, Aida Razi}

Department of Anatomy and Cell Biology, McGill University, Montreal. Canada.

Our research aims to understand the structure and function of new antimicrobial targets, developing them into tangible enzymes for intervention with new antibiotics. Current work focuses on the assembly process of the ribosome; a pathway of tremendous potential but that has not been explored as a target for antimicrobials yet. In recent years, or group and others found that the final steps in the ribosome assembly process are dedicated to the maturation of the functional sites [1-3]. Several protein factors bind at or near these sites ensuring they fold into their functional conformation. However, their specific functions or how these assembly factors assist maturation are still unknown [4]. Introduction of electron detectors in cryo-EM two years ago has revolutionized structural biology [5]. We are using these detectors to obtain structures of assembly intermediates alone and in complex with assembly factors to atomic resolution [6,7]. These structures allow for amino acid level description of the molecular mechanism and the conformational changes that the assembly intermediates and ribosomal particles undergo upon interaction. This is critical information to fully understand the function of these assembly factors in the process of assembly of the ribosome and thus, for the development of new antibiotics targeting them. Overall, this work is impacting our ability to treat infectious diseases.

The ribosome is the enzyme responsible for protein synthesis and the most complex macromolecule existing in bacterial cells. In Escherichia coli the ribosome is made from more than 50 different components organized into the small (30S) and large (50S) ribosomal subunits. Understanding how the components of the bacterial ribosome come together and organize themselves still remains a daunting challenge. In spite of its complexity, each bacterial cell assembles more than 20,000 ribosomes in less than 30 minutes. Cells are able to maintain this assembly rate because a number of auxiliary factors make the process extremely efficient. Our work focuses on the YjeQ GTPase assembly factor. Recent work indicates that this protein assists the late stages of assembly of the 30S subunit. To gain new key insights regarding the function of YjeQ in the context of the mature 30S subunit, we have obtained the $3 \mathrm{D}$ structure of the $30 \mathrm{~S}+\mathrm{YjeQ}$ complex at $\sim 5 \AA$ resolution using an electron microscope equipped with a direct electron detector. The cryo-EM map shows that YjeQ binds to the decoding center. The structure shows that YjeQ anchors tightly to the three domains of the subunit, mainly through its $\mathrm{N}$ - and Cterminal domains. The OB-fold contacts the body of the $30 \mathrm{~S}$ subunit whereas the zinc-finger domain anchors the protein to both the head and platform in the particle. The GTPase domain almost completely covers the decoding center and contacts the platform through a long loop. In addition, we obtained the cryo-EM map of free 30S mature subunit at comparable resolution allowing us to visualize the structure of this particle when it is not confined in a crystal lattice. Surprisingly, we found that a long stretch of helix 44 is unstructured and does not adopt the conformation described by the $30 \mathrm{~S}$ subunit X-ray structure. However, binding of YjeQ to the free 30S subunit stabilizes helix 44 into a conformation that is similar to that on the X-ray structure. The specific interactions between the OB-fold of YjeQ and helix 44 depicted in the cryo-EM map indicates that YjeQ has a role as a checkpoint protein dedicated to test the decoding fidelity of the $30 \mathrm{~S}$ subunit before the particle is release to the pool of actively translating ribosomes. Checkpoint functions have been described for eukaryotic ribosome assembly factors. However, this work provides the first example of a bacterial assembly factor that tests a specific 
translation mechanism of the $30 \mathrm{~S}$ subunit.

References:

[1] Jomaa A, Stewart G, Martin-Benito J, et al. RNA 2011; 17(4): 697-709.

[2] Li N, Chen Y, Guo Q, et al. Nucleic Acids Res 2013; 41(14): 7073-83.

[3] Jomaa A, Jain N, Davis JH, et al. Nucleic Acids Res 2014; 42(5): 3419-35.

[4] Shajani Z, Sykes MT, Williamson JR. Annu Rev Biochem 2011; 80: 501-26.

[5] Bai XC, McMullan G,Scheres SH. Trends Biochem Sci 2015; 40(1): 49-57.

[6] Ni X, Davis JH, Jain N, et al. Nucleic Acids Res 2016; 44(17): 8442-55.

[7] Zhang X, Yan K, Zhang Y, et al. Nucleic Acids Res 2014; 42(21): 13430-9.

[8] He K, Ludtke SJ, Wu Y, et al. Biophys Chem 1994; 49(1): 83-9.
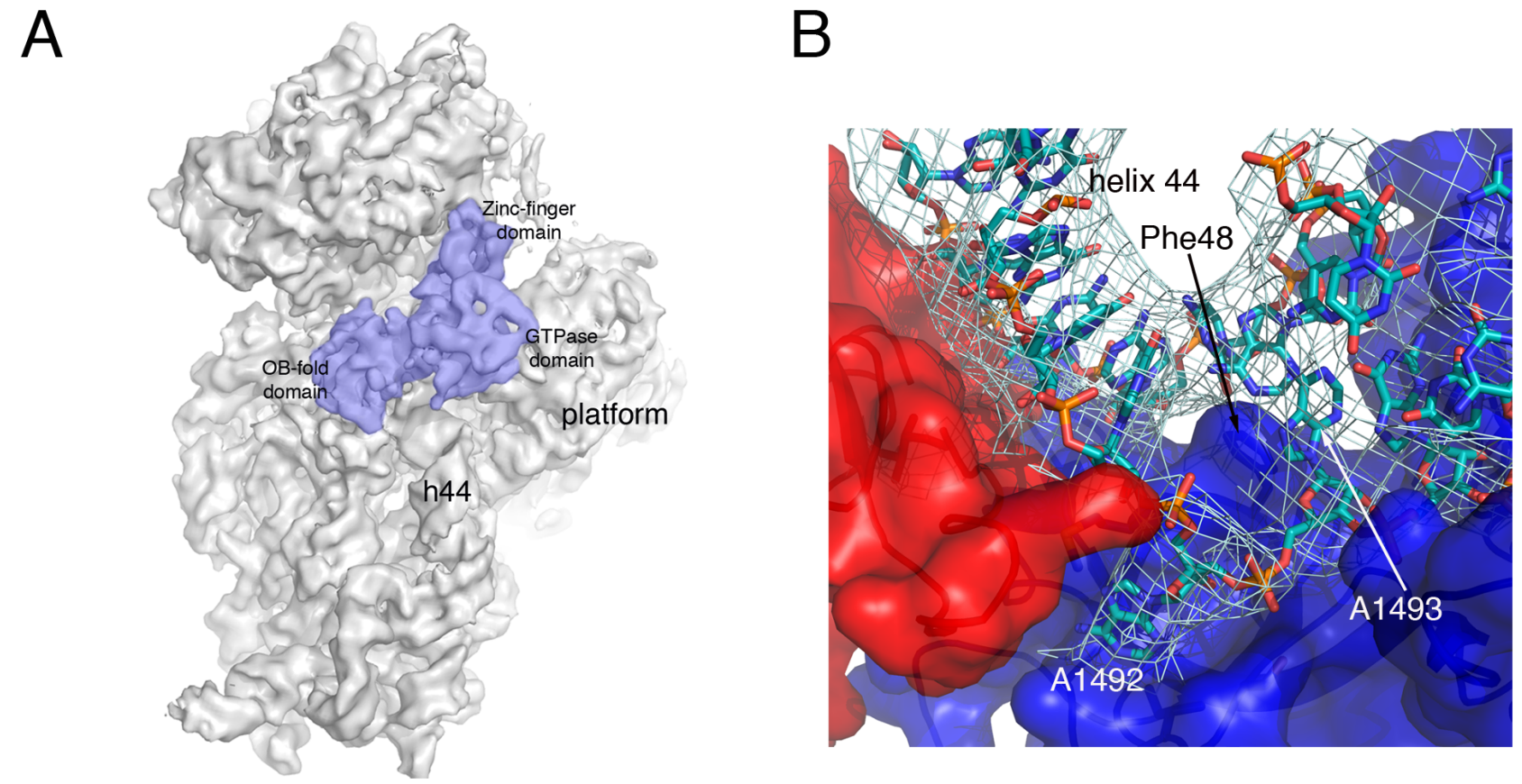

Figure 1. Cryo-EM structure of the $30 \mathrm{~S}+\mathrm{YjeQ}$ complex. (A) Front view of the $30 \mathrm{~S}+\mathrm{YjeQ}$ complex cryo-EM structure. (B) YjeQ checkpoint role in ribosome fidelity. Atomic model fitted into the cryo-EM density map showing that A1492 on the $16 \mathrm{~S}$ rRNA adopts a flip out conformation. 